\title{
Penser la musique à l'ère du web (2)
}

Issus de la conférence internationale «Penser la musique à l'ère du web/Thinking Music in the Web Age $»^{2}$, les articles de ce numéro de De musica poursuivent l'enquête amorcée dans le numéro précédent sur les transformations rapides et profondes de la culture musicale contemporaine à l'ère du web. Ils traitent notamment de quelques aspects significatifs de l'impact de la révolution numérique sur la production, la diffusion et la réception de la musique.

Un premier groupe d'articles porte sur la production musicale. Les nouvelles technologies, et en particulier les développements récents de l'intelligence artificielle et de la communication télématique, mettent en effet de nouveaux outils à la disposition de la créativité des interprètes et des compositeurs, au-delà des instruments acoustiques et électroniques et au-delà des possibilités offertes par l'écriture des partitions. D'une part, de nouveaux types de production musicale collaborative sont rendus possibles par des appareils portables et très courants tels que les smartphones (Le Bouteiller) et par le moyen de logiciels novateurs (Guesdon \& Le Guern); d'autre part, les nouvelles technologies amplifient et renouvellent les possibilités créatrices traditionnelles, comme l'exploitation compositionnelle des archives sonores (Cossettini) - une ressource aujourd'hui renforcée par la capillarité et la vitesse fulgurante de la diffusion par l'Internet - et les pratiques d'improvisation, qui peuvent désormais s'étendre à l'interaction par des réseaux informatiques et avec des performers computationnels (Bertinetto).

Un second groupe d'articles examine les mutations qui ont affecté les systèmes de diffusion et de réception de la musique. Dans un contexte désormais largement dominé par l'offre des plateformes de musique en streaming - une formule qui semble avoir rendu inactuelles les stratégies de

\footnotetext{
${ }^{2}$ Voir De Musica 24/2.
} 
vente fondées sur le téléchargement - il est intéressant de réfléchir sur les destins de la production discographique, que ce soit dans le cas du CD ou du vinyle, un format qui a connu une renaissance inattendue (Lephay), mais aussi sur la façon dont ces mêmes plateformes ont contribué à redéfinir la figure du mélomane, lié plus particulièrement au monde de l'opéra (Pallazzetti) ou à la transformation des pratiques sociales de la flânerie et du collectionnisme (Ruta). Une réflexion particulière a enfin été consacrée à l'introduction des technologies fondées sur le «blockchain » (Magaudda), dont les avantages sur le plan de l'organisation des traces musicales sont peut-être moins évidents que ce que l'on pouvait espérer.

Riche et varié, l'ensemble des contributions de ce double numéro de De Musica (24, II and 25, I) est loin d'épuiser l'argument que nous nous sommes proposés d'explorer. Mentionnons, parmi les aspects qui auraient sans doute mérité une plus grande attention, les nouvelles formes de production audiovisuelle qui caractérisent la diffusion de la musique aujourd'hui, de plus en plus vouée à combiner sons et images, et la manière dont la technologie numérique a favorisé l'émergence de nouveaux genres musicaux. Nous espérons quand même que ce premier tour d'horizon pourra offrir au lecteur quelques éléments de réflexion sur les mutations qui ont affecté le monde musical à une époque que nous avons choisi de présenter sous la bannière du web. Car cet «hypermédia », dans lequel nous baignons désormais depuis une bonne trentaine d'années, et dont la crise sanitaire mondiale a peut-être encore amplifié le pouvoir, nous a imposé une profonde révision de nos manières de penser la musique. 